\title{
Can Trend Followers Survive in the Long-Run? Insights from Agent-Based Modeling
}

\author{
Xue-Zhong $\mathrm{He}^{1 *}$, Philip Hamill ${ }^{2}$, and Youwei $\mathrm{Li}^{3 \dagger}$ \\ 1 School of Finance and Economics, University of Technology, Sydney, PO Box \\ 123 Broadway, NSW 2007, Australia. tony.he1@uts.edu.au \\ 2 School of Business, Retail and Financial Services, University of Ulster, \\ Coleraine, Cromore Road, County Derry, BT52 1SA, United Kingdom. \\ pa.hamill@ulster.ac.uk \\ 3 School of Management and Economics, Queen's University Belfast, 25 University \\ Square, Belfast, BT7 1NN, United Kingdom. y.li@qub.ac.uk
}

Summary. This paper uses a simple stochastic market fraction (MF) asset pricing model to investigate market dominance, profitability, and how traders adopting fundamental analysis or trend following strategies can survive under various market conditions in the long/short-run. This contrasts with the modern theory of finance which relies on the paradigm of utility maximizing representative agents and rational expectations assumptions which some contemporary theorists regard as extreme. This school of thought would predict that trend followers will be driven out of the markets in the long-run. Our analysis shows that in a MF framework this is not necessarily the case and that trend followers can survive in the long-run.

\section{Introduction}

The modern theory of finance relies on the paradigm that asset prices are the outcome of the market interaction of utility maximizing representative agents who are rational when forming expectations about future market outcomes. The assumption that agents rationally impound all relevant information into their trading decisions produces price changes which are random, and consequently exhibit random walk behaviour. The representative agent assumption, as argued by Friedman [14], leads to the conjecture that irrational traders (also called less informed traders or chartists) could profit in the short-run, but are expected to perish in the long-run while rational traders (also called informed traders or fundamentalists) should be the only long-run survivors.

\footnotetext{
* Acknowledgement: Xue-Zhong He would like to thank the financial support from the Australian Research Council (ARC) under discovery grant (DP0450526), and the University of Technology, Sydney under a Research Excellence Grant (REG).

$\dagger$ Corresponding author.
} 
Despite all the evidence presented in academic journals that security prices follow random walks the use of technical trading rules is widespread amongst financial market practitioners as evidenced by the growth in hedge funds employing quantitative trading strategies: trend following is one of the most popular trading rules. Also, finance theory provides limited economic explanations for the well documented stylized facts reported in the empirical finance literature (see Pagan [30]). The existence of excess volatility (asset return is more volatile relative to the dividends and underlying cash flows), volatility clustering (high/low asset return fluctuations are followed by high/low fluctuations), either positive or negative skewness and excess kurtosis (compared to normally distributed returns), and long-range dependence (insignificant autocorrelations (ACs) of raw returns and hyperbolic decline of ACs of the absolute and squared returns, see Ding et al.[13]) are difficult to accommodate within the established theoretical structure of market efficiency and rational expectations (see, for example, Shiller [31]). In practice, GARCH methodology has been successful modeling volatility clustering and capturing the short-run dynamics of volatility, but fails to provide an economic explanation.

Agent-Based Modeling is an alternative paradigm which may provide an appropriate theoretical and methodological framework to explain the stylized facts. For those new to this area we refer them to the survey papers by Hommes [20] and LeBaron [24] for the recent development in this literature. In contrast to the traditional assumptions of investor homogeneity and rational expectations, the latter of which is regarded as an extreme informational assumption (see, for example, Cochrane [11]), agent-based models allow for heterogeneous agents, potentially showing bounded rational behaviour, who have different attitudes to risk and different expectations about the future evolution of prices. This approach has been shown to be able to characterize the dynamics of financial asset returns. The works of Arthur et al. [2], Brock and Hommes [3], Chiarella [6], Chiarella and He [7] and [8], Day and Huang [12] LeBaron [21], Levy et al. [25], Lux [28], among others, are examples of this approach. Agent-Based Models attempt to explain various types of market behaviour and to replicate the well documented empirical features of actual financial markets.

Heterogeneous agent-based models have had success in explaining market behavior and reproducing stylized facts. However, there are few works explicitly investigating if irrational traders can survive in the long-run. In this paper a market fractions (MF) model with heterogeneous traders - fundamentalists (rational investors who believe the market price is mean reverting to fundamental price) and trend followers (irrational investors who believe market price will follow the trend generated from historical prices) - participate in a simple stochastic asset-pricing and wealth dynamics framework to investigate market dominance, profitability, and if they survive in the short/long-run. Although the techniques discussed in Arnold [1] may be useful for analyzing the stochastic model the mathematical analysis of nonlinear stochastic dynamical systems is difficult in general. Therefore, this analysis is conducted through 
Monte Carlo simulation (see Li et al. [26] and [27] for a more systematic study on this). The results from our simulation analysis show that, as expected, fundamentalists survive in the long-run and their profitability improves as they become increasingly confident of their forecasts of fundamental value. More interestingly, the key insight from our analysis is that trend followers can survive in the long-run even though they don't engage in information processing to establish the fundamental value of the asset to inform their trading strategy. In the context of our MF model this can be explained by the learning mechanism encapsulated in the trend followers forecasts. Our results also show that trend followers' profitability increases as their market share increases and when fundamentalists become naive traders.

This paper is organized as follows. Section 2 outlines a market fraction model with heterogeneous agents. In this model the market clearing price is set by a market maker who adjusts the market price in response to aggregate excess demand in the market. Next, the expectations and learning mechanisms for the fundamentalists and trend followers is introduced. The latter part of this section develops the full market fraction stochastic model for asset prices and wealth dynamics. In Section 3 the profitability and survivability of fundamental and trend following strategies is explored under alternative scenarios. Section 4 discusses the key insights from the study and identifies logical extensions for future research.

\section{Heterogeneous Beliefs and Market Fractions}

Intuitively, market population fractions among different types of traders play an important role in financial markets. Markets can be driven by certain types of investors at different time periods. This is particularly the case in either a bull or bear market. Empirical evidence from Taylor and Allen [32] suggests that at least $90 \%$ of the traders place some weight on technical analysis, such as moving average and trend following rules, over various time horizons. In particular, traders rely more on technical analysis, as opposed to fundamental analysis, at shorter horizons. As the time horizons increases more traders rely on fundamental rather than technical analysis. In addition, there are a proportion of traders who do not change their strategies over all time horizons. This situation is consistent with money-managers following a longer-term value investing strategy. Their time horizon for realizing gains is often years as opposed to days for the technical analyst, see Haugen [16] and Chan and Lakonishok [5]. Theoretically, the study by Brock and Hommes [3] shows that when different groups of traders have different expectations about future prices and dividends compete between trading strategies and choose their strategy according to an evolutionary fitness measure the corresponding deterministic system can exhibit very complicated, and even, chaotic dynamics. The adaptive switching mechanism proposed by Brock and Hommes [3] 
is an important element of the adaptive belief model. It is based on both a fitness function and a discrete choice probability.

In this paper we employ the market fraction $(\mathrm{MF})$ model introduced in $\mathrm{He}$ and $\mathrm{Li}$ [17]. It is a simplified version of Brock and Hommes' framework which assumes that the market fraction parameters among heterogeneous agents are fixed. Apart from its mathematical tractability this simplification has a number of distinct advantages. First, it clearly identifies how different market fractions influence the market price. In Brock and Hommes' framework this is difficult due to the amplifying effect of the exponential function used in the discrete choice probability which makes the market fractions very sensitive to price changes and the stated fitness functions. Second, our model doesn't allow agents to switch between trading strategies. This makes it easier to characterize market dominance, profitability, and survivability. Finally, it is important to understand how the trading strategies are linked to aspects of price behaviour. Having market fractions among heterogeneous agents as fixed parameters allows for an explicit examination of how market fractions influence price behaviour.

The MF model considered in the following discussion, introduced in $\mathrm{He}$ and $\mathrm{Li}$ [17], follows the standard discounted value asset pricing model with heterogeneous agents. The market clearing price is arrived at via a market maker scenario in line with Day and Huang [12] and Chiarella and He [10] rather than the Walrasian auctioneer scenario used in Brock and Hommes [4]. We focus on a simple case in which there are three classes of participants in the asset market: two groups of traders, fundamentalists and trend followers, and a market maker.

\subsection{Market Fraction and Market Maker}

Consider an asset pricing model with one risky asset and one risk-free asset. It is assumed that the supply of the risk-free asset is perfectly elastic with a gross return of $R=1+r / K$, where $r$ stands for a constant risk-free rate per annum and $K$ stands for the trading frequency. Typically, $K=1,12,52$ and 250 for trading periods of a year, month, week, and a day. To calibrate the stylized facts observed from daily price movements in financial markets $K$ is set equal to 250 .

Let $P_{t}$ be the price (ex-dividend) per share for the risky asset at time $t$ and $\left\{D_{t}\right\}$ be the stochastic dividend process for the risky asset. Then the wealth of a typical investor $h$ at $t+1$ is given by

$$
W_{h, t+1}=R W_{h, t}+\left[P_{t+1}+D_{t+1}-R P_{t}\right] z_{h, t},
$$

where $W_{h, t}$ and $z_{h, t}$ are the wealth and the number of shares of the risky asset purchased by investor $h$ at $t$. Let $E_{h, t}$ and $V_{h, t}$ be the beliefs of type $h$ traders about the conditional expectation and variance of quantities at $t+1$ based on their information set at time $t$. Where the excess capital gain on the risky asset at $t+1$ is denoted by $R_{t+1}$, that is 


$$
R_{t+1}=P_{t+1}+D_{t+1}-R P_{t}
$$

Then it follows from (1) and (2) that

$$
E_{h, t}\left(W_{t+1}\right)=R W_{t}+E_{h, t}\left(R_{t+1}\right) z_{h, t}, \quad V_{h, t}\left(W_{t+1}\right)=z_{h, t}^{2} V_{h, t}\left(R_{t+1}\right),
$$

where $z_{h, t}$ is the demand by agent $h$ for the risky asset. Assume that trader $h$ has a constant absolute risk aversion (CARA) utility function with the risk aversion coefficient $a_{h}$ (that is $U_{h}(W)=-e^{-a_{h} W}$ ), the optimal demand $z_{h, t}$ for the risky asset is determined by maximizing the expected utility of wealth, that is

$$
z_{h, t}=\frac{E_{h, t}\left(R_{t+1}\right)}{a_{h} V_{h, t}\left(R_{t+1}\right)} .
$$

Given the heterogeneity and the nature of asymmetric information among traders we consider two trading strategies corresponding to two types of boundedly rational traders: fundamentalists and trend followers. Their beliefs are defined in the following discussion. Assume the market fraction of the fundamentalists and trend followers is $n_{1}$ and $n_{2}$ with risk aversion coefficient $a_{1}$ and $a_{2}$, respectively. Let $m=n_{1}-n_{2} \in[-1,1]$. Obviously, $m=1,-1$ correspond to the cases when all the traders are fundamentalists or trend followers. Assume a zero supply of outside shares. Then, using (4), the aggregate excess demand per investor $\left(z_{e, t}\right)$ is given by

$$
z_{e, t} \equiv n_{1} z_{1, t}+n_{2} z_{2, t}=\frac{1+m}{2} \frac{E_{1, t}\left[R_{t+1}\right]}{a_{1} V_{1, t}\left[R_{t+1}\right]}+\frac{1-m}{2} \frac{E_{2, t}\left[R_{t+1}\right]}{a_{2} V_{2, t}\left[R_{t+1}\right]} .
$$

To complete the model we assume that the market is cleared by a market maker. The role of the market maker is to take a long (when $z_{e, t}<0$ ) or short (when $z_{e, t}>0$ ) position so as to clear the market. At the end of period $t$, after the market maker has carried out all transactions, he or she adjusts the price for the next period in the direction of the observed excess demand. Let $\mu$ be the speed of price adjustment of the market maker. To capture unexpected market news or noise created by noise traders we introduce a noisy demand term $\tilde{\delta}_{t}$ which is an IID normally distributed random variable with $\tilde{\delta}_{t} \sim \mathcal{N}\left(0, \sigma_{\delta}^{2}\right)$. In this paper, we assume a constant volatility noisy demand and the volatility is related to an average fundamental price level. This noisy demand may also depend on the market price. Theoretically, how the price dynamics are influenced by adding different noisy demand is still a difficult problem. Here, we focus on the constant volatility noisy demand case and use Monte Carlo simulations and statistical analysis to gain some insights into this problem. Based on these assumptions the market price is determined by

$$
P_{t+1}=P_{t}+\mu z_{e, t}+\tilde{\delta}_{t} .
$$

Using (5), this becomes

$$
P_{t+1}=P_{t}+\frac{\mu}{2}\left[(1+m) \frac{E_{1, t}\left[R_{t+1}\right]}{a_{1} V_{1, t}\left[R_{t+1}\right]}+(1-m) \frac{E_{2, t}\left[R_{t+1}\right]}{a_{1} V_{2, t}\left[R_{t+1}\right]}\right]+\tilde{\delta}_{t} .
$$


We use Figure 1 to illustrate the general role of heterogeneous expectation for our two groups of agents and how the market cleaning price is arrived at. Let $p_{t, t+1}^{f, e}$ and $p_{t, t+1}^{c, e}$ be the expected price at time $t+1$ for the fundamentalists and chartists conditional upon their information set at time $t$. The market maker aggregates the demand from agents' heterogeneous expectations to form the expected market price $p_{t, t+1}^{e}$ at time $t$ and adjusts the market price at time $t+1$ accordingly. The important feature of this structure is that the price generating mechanism is driven by expectations feedback. Observed market prices are then used to form expectations for the next period which in turn feeds back into the price generating process.

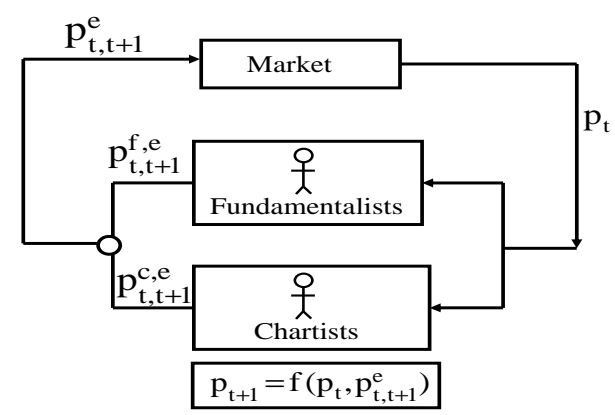

Fig. 1. Expectations Feedback

It should be pointed out that market maker behaviour in this model is highly stylized. For instance, the inventory of the market maker built up as a result of the accumulation of various long and short positions is not considered. This could affect his or her behaviour and the market maker price setting role in (7) could be a function of the inventory. Allowing $\mu$ to be a function of inventory would be one way to model such behaviour. In this paper it is best thought of as a market friction. One of the aims of our analysis is to understand how this friction affects the market dynamics.

\subsection{Fundamentalists}

We assume that $F_{t}=\left\{P_{t}, P_{t-1}, \cdots, D_{t}, D_{t-1}, \cdots\right\}$ is the common information set at time $t$. Apart from the common information set, the fundamentalists have superior information on the fundamental value, $P_{t}^{*}$, of the risky asset and they also realize the existence of non-fundamental traders such as trend followers to be introduced in the following discussion. They believe that the stock price may be driven away from the fundamental value in the short-run, but it will eventually converge to the fundamental value in the long-run. The speed of convergence measures their confidence level in the fundamental value. More precisely, we assume that the fundamental value satisfies a stationary 
random walk process (as we know that the fundamental value driven by this random walk process can be negative)

$$
P_{t+1}^{*}=P_{t}^{*}\left[1+\sigma_{\epsilon} \tilde{\epsilon}_{t}\right], \quad \tilde{\epsilon}_{t} \sim \mathcal{N}(0,1), \quad \sigma_{\epsilon} \geq 0, \quad P_{0}^{*}=\bar{P}>0,
$$

where $\tilde{\epsilon}_{t}$ is independent of the noisy demand process $\tilde{\delta}_{t}$. We assume the conditional mean and variance of the fundamental traders are

$$
E_{1, t}\left(P_{t+1}\right)=P_{t}+\alpha\left(P_{t+1}^{*}-P_{t}\right), \quad V_{1, t}\left(P_{t+1}\right)=\sigma_{1}^{2},
$$

where $\sigma_{1}^{2}$ stands for a constant variance for the price. Here parameter $\alpha \in[0,1]$ is the speed of price adjustment toward the fundamental value. It measures their level of confidence in fundamental value. Specifically, for $\alpha=1$ the fundamental traders are fully confident about the fundamental value and adjust their expected price at the next period instantaneously to the fundamental value. When $\alpha=0$ fundamentalists become naive traders. In general, the fundamental traders believe that markets are efficient and prices converge to their fundamental value. An increase (decrease) in $\alpha$ indicates that the fundamental traders have high (low) confidence in their estimated fundamental value, leading to a quick (slow) adjustment of the expected price towards the fundamental price.

\subsection{Trend followers}

Unlike the fundamentalists, trend followers are technical traders who believe that future price changes can be predicted from various patterns or trends generated from the history of prices. The trend followers are assumed to extrapolate the latest observed price change over prices' long-run sample mean and to adjust their variance estimate accordingly. More precisely, their conditional mean and variance are assumed to satisfy

$$
E_{2, t}\left(P_{t+1}\right)=P_{t}+\gamma\left(P_{t}-u_{t}\right), \quad V_{2, t}\left(P_{t+1}\right)=\sigma_{1}^{2}+b_{2} v_{t},
$$

where $\gamma, b_{2} \geq 0$ are constants, and $u_{t}$ and $v_{t}$ are the sample mean and variance, respectively, which follow a learning process. The parameter $\gamma$ measures the extrapolation rate. High (low) values of $\gamma$ correspond to strong (weak) extrapolation from the trend. The coefficient $b_{2}$ measures the influence of the sample variance on the conditional variance estimated by the trend followers who believe in a more volatile price movement. Various learning schemes can be used to estimate the sample mean $u_{t}$ and variance $v_{t}$ (see Chiarella and $\mathrm{He}([9],[10])$ for related studies on heterogeneous learning and asset pricing models with heterogeneous agents who's conditional mean and variance follow various learning processes). In this paper we assume that

$$
\begin{aligned}
& u_{t}=\delta u_{t-1}+(1-\delta) P_{t}, \\
& v_{t}=\delta v_{t-1}+\delta(1-\delta)\left(P_{t}-u_{t-1}\right)^{2},
\end{aligned}
$$


where $\delta \in[0,1]$ is a constant. This is the limiting process of a geometric decay process where the memory lag length tends to infinity. Basically, a geometric decay probability process $(1-\delta)\left\{1, \delta, \delta^{2}, \cdots\right\}$ is associated with historical prices $\left\{P_{t}, P_{t-1}, P_{t-2}, \cdots\right\}$. The parameter $\delta$ measures the geometric decay rate. For $\delta=0$, the sample mean $u_{t}=P_{t}$, which is the latest observed price, while $\delta=0.1,0.5,0.95$ and 0.999 gives a half life of 0.43 day, 1 day, 2.5 weeks and 2.7 years, respectively. The selection of this process is two fold. First, traders tend to put a high weight on the most recent prices and less weight on the more remote prices when they estimate the sample mean and variance. Second, it has the mathematical advantage of analytical tractability.

\subsection{The Complete Stochastic Model}

To simplify our analysis we assume that the dividend process $D_{t}$ follows a normal distribution $D_{t} \sim \mathcal{N}\left(\bar{D}, \sigma_{D}^{2}\right)$. The expected long-run fundamental value $\bar{P}=\bar{D} /(R-1)$ and the unconditional variances of price and dividend over the trading period are related by $\sigma_{D}^{2}=q \sigma_{1}^{2}$. In this paper, we choose $\sigma_{1}^{2}=\sigma_{\bar{P}}^{2} / K$ and $q=r^{2}$. This can be justified as follows. Let $\sigma_{\bar{P}}$ be the annual volatility of $P_{t}$ and $\bar{D}_{t}=r P_{t}$ be the annual dividend. Then the annual variance of the dividend $\bar{\sigma}_{D}^{2}=r^{2} \sigma_{\bar{P}}^{2}$. Therefore $\sigma_{D}^{2}=\bar{\sigma}_{D}^{2} / K=r^{2} \sigma_{\bar{P}}^{2} / K=r^{2} \sigma_{1}^{2}$. For all numerical simulations in this paper we choose $\bar{P}=\$ 100, r=5 \%$ p.a. $\sigma=20 \%$ p.a., $K=250$. Correspondingly, $R=1+0.05 / 250=1.0002, \sigma_{1}^{2}=(100 \times 0.2)^{2} / 250=8 / 5$ and $\sigma_{D}^{2}=1 / 250$. Based on assumptions (9)-(10), the fundamentalists' optimal demand is

$$
z_{1, t}=\frac{1}{a_{1}(1+q) \sigma_{1}^{2}}\left[\alpha\left(P_{t+1}^{*}-P_{t}\right)-(R-1)\left(P_{t}-\bar{P}\right)\right] .
$$

In particular, when $P_{t}^{*}=\bar{P}$,

$$
z_{1, t}=\frac{(\alpha+R-1)\left(\bar{P}-P_{t}\right)}{a_{1}(1+q) \sigma_{1}^{2}} .
$$

Similarly, from (10), (using $\bar{D}=(R-1) \bar{P})$ the trend followers' optimal demand is

$$
z_{2, t}=\frac{\gamma\left(P_{t}-u_{t}\right)-(R-1)\left(P_{t}-\bar{P}\right)}{a_{2} \sigma_{1}^{2}\left(1+q+b v_{t}\right)},
$$

where $b=b_{2} / \sigma_{1}^{2}$. Subsisting (13) and (15) into (7), the price dynamics under a market maker is determined by the following 4-dimensional stochastic difference system (SDS hereafter) 


$$
\left\{\begin{aligned}
P_{t+1}= & P_{t}+\frac{\mu}{2}\left[\frac{1+m}{a_{1}(1+q) \sigma_{1}^{2}}\left[\alpha\left(P_{t+1}^{*}-P_{t}\right)-(R-1)\left(P_{t}-\bar{P}\right)\right]\right. \\
& \left.\quad+(1-m) \frac{\gamma\left(P_{t}-u_{t}\right)-(R-1)\left(P_{t}-\bar{P}\right)}{a_{2} \sigma_{1}^{2}\left(1+q+b v_{t}\right)}\right]+\tilde{\delta}_{t}, \\
u_{t}= & \delta u_{t-1}+(1-\delta) P_{t}, \\
v_{t}= & \delta v_{t-1}+\delta(1-\delta)\left(P_{t}-u_{t-1}\right)^{2}, \\
P_{t+1}^{*}= & P_{t}^{*}\left[1+\sigma_{\epsilon} \tilde{\epsilon}_{t}\right] .
\end{aligned}\right.
$$

Using Monte Carlo simulation and statistical analysis, He and Li [17] found that the long-run behaviour and convergence of the market prices and various under and over-reaction autocorrelation patterns of returns can be characterized by the dynamics, including the stability and bifurcations, of the underlying deterministic system. In the relation to the ability of the MF model to characterize the stylized facts, especially the long-range dependence in volatility, He and Li [18] demonstrate that agent heterogeneity, risk-adjusted trend chasing through the geometric learning process, and the interplay of noisy fundamental and demand processes and the underlying deterministic dynamics can be the source of power-law distributed fluctuations. In particular, the noisy demand plays an important role in the generation of insignificant autocorrelations (ACs) on returns, while the significant decaying $\mathrm{AC}$ patterns of the absolute returns and squared returns are more influenced by the noisy fundamental process. A statistical analysis based on Monte Carlo simulations is conducted to characterize the decay rate. Realistic estimates of the power-law decay indices and the (FI)GARCH parameters are presented. This analysis provides some insights into the understanding of financial markets. In the following discussion we introduce measures of wealth dynamics and we explore the potential of the MF model to characterize the profitability and survivability of fundamental and trend following strategies.

\subsection{Wealth Dynamics and Shares}

We assume that traders' wealth follows a stochastic process. To be able to measure the wealth dynamics among different trading strategies and to examine the market dominance and price behaviour we introduce two wealth measures. The first measures the absolute level of the wealth share (or proportion) of the representative agent from each type, called the absolute wealth share for short, which is defined by

$$
w_{1, t}=\frac{W_{1, t}}{W_{1, t}+W_{2, t}}, \quad w_{2, t}=\frac{W_{2, t}}{W_{1, t}+W_{2, t}},
$$

where $W_{1, t}$ and $W_{2, t}$ are the wealth at time $t$ of the representative trader of the fundamentalists and trend followers, respectively. This measure can be used to measure the evolutionary performance or profitability of the two trading strategies: As $w_{1, t}\left(w_{2, t}\right)$ increases the profitability of the fundamentalists 
(trend followers) increases. The second measures the overall market wealth share, called the market wealth share for short, of the different trading strategies. It is defined as the market fraction weighted average of the absolute wealth proportions,

$$
\bar{w}_{1, t}=\frac{(1+m) W_{1, t}}{(1+m) W_{1, t}+(1-m) W_{2, t}}, \quad \bar{w}_{2, t}=\frac{(1-m) W_{2, t}}{(1+m) W_{1, t}+(1-m) W_{2, t}}
$$

A high market wealth share $\bar{w}_{1, t}\left(\bar{w}_{2, t}\right)$ indicates market dominance of the fundamentalists (trend followers) with respect to the overall market wealth. Let $V_{1, t}=1 / W_{1, t}$ and $V_{2, t}=1 / W_{2, t}$. Then it follows from (1) that

$$
V_{1, t+1}=\frac{V_{1, t}}{R+R_{t+1} z_{1, t} V_{1, t}}, \quad V_{2, t+1}=\frac{V_{2, t}}{R+R_{t+1} z_{1, t} V_{2, t}} .
$$

Note that

$$
\begin{aligned}
& \frac{V_{1, t}}{V_{1, t}+V_{2, t}}=\frac{1 / W_{1, t}}{1 / W_{1, t}+1 / W_{2, t}}=\frac{W_{2, t}}{W_{1, t}+W_{2, t}} \\
& \frac{V_{2, t}}{V_{1, t}+V_{2, t}}=\frac{1 / W_{2, t}}{1 / W_{1, t}+1 / W_{2, t}}=\frac{W_{1, t}}{W_{1, t}+W_{2, t}}
\end{aligned}
$$

and, therefore, the absolute wealth shares are determined by

$$
w_{1, t}=\frac{V_{2, t}}{V_{1, t}+V_{2, t}}, \quad w_{2, t}=\frac{V_{1, t}}{V_{1, t}+V_{2, t}}
$$

and the market wealth shares are governed by

$$
\bar{w}_{1, t}=\frac{(1+m) V_{2, t}}{(1+m) V_{2, t}+(1-m) V_{1, t}}, \bar{w}_{2, t}=\frac{(1-m) V_{1, t}}{(1+m) V_{2, t}+(1-m) V_{1, t}} .
$$

For these wealth measures it is difficult to obtain explicit closed form expressions in terms of (stationary) state variables. In this paper we use the auxiliary functions $\left(V_{1, t}, V_{2, t}\right)$ and numerical simulations to study the wealth dynamics of the fundamentalists and trend followers and the market impact of the two different trading strategies.

\section{Wealth Accumulation, Profitability and Survivability}

Friedman [14] argued that irrational traders (such as the trend followers in our model) may do better than rational traders (such as the fundamentalists) in the short-run, but over the long-run they will be driven out of the market and rational traders will be the only long-run survivors. We now justify Friedman's hypothesis by analyzing the wealth dynamics of our heterogeneous market fraction model in which traders' beliefs are time invariant. Consequently, we examine profitability and survivability of both types of trading strategies. The dynamics of the speed of price adjustment and the market fraction are considered in the following discussion. 


\subsection{Dynamics of the Price Adjustment Speed $\alpha$}

In our model, the market price is related to the fundamental price through the activity from the fundamentalists. Therefore, how the market price reflects the fundamental price depends on the reaction of the fundamentalists to the fundamental price. It is believed that chartists may perform better when the market price is far away from the fundamental price, while the fundamentalists may do better when the market price reflect the fundamental price. Hence it is interesting to analyze the wealth dynamics when the fundamentalists behave differently. In the following we examine the wealth dynamics of the model when the confidence level of the fundamentalists on the fundamental price, measured by parameter $\alpha$, changes.

To assess the impact of $\alpha$ we choose parameters set to

$$
\gamma=2.1, \delta=0.85, \mu=0.43, m=0, w_{1,0}=0.5, \alpha=1,0.5,0.1,0
$$

by fixing market fraction $m$ and varying $\alpha$. For each set of parameters, we run one simulation over 20,000 time periods in order to see possible limiting behaviours.

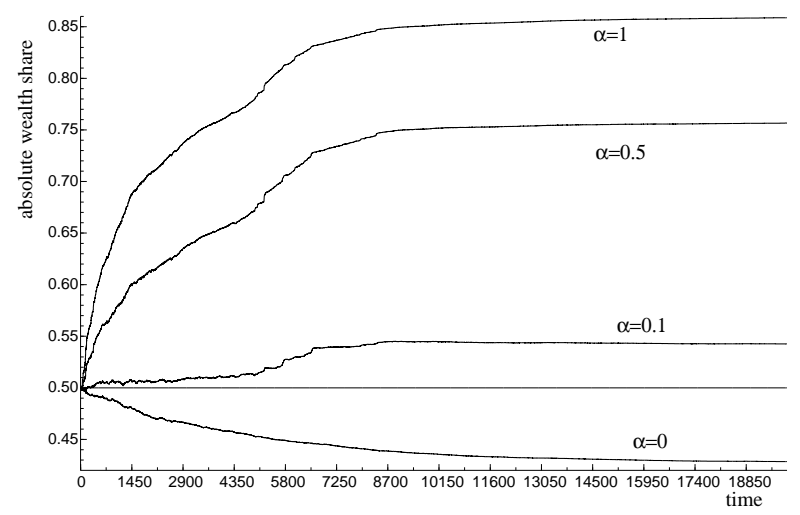

Fig. 2. Time series of the absolute wealth accumulation of the fundamentalists $w_{1, t}$ with $\alpha=1,0.5,0.1$ and 0 .

Fig. 2 demonstrates the absolute wealth share accumulations for the fundamentalists with $\alpha=1,0.5,0.1,0$ and keeping all the other conditions the same. This figure shows that (i) trend followers survive in the long-run for $\alpha=1,0.5$ and 0.1 in the sense that their absolute wealth share does not vanish, although they accumulate less wealth shares over the time period; (ii) the trend followers do better than the fundamentalists when $\alpha=0$; (iii) the profitability of the fundamentalists improves as $\alpha$ increases (i.e. as they become more confident in their estimated fundamental value). For $\alpha=0$, the absolute wealth share of the fundamentalists is dropped from $50 \%$ to about $43 \%$, while 
for $\alpha=0.1,0.5$ and 1 , it is increased from $50 \%$ to about $55 \%, 76 \%$ and $86 \%$, respectively. These results are further confirmed when we run Monte Carlo simulations. We ran 1,000 independent simulations and discard the first 1,000 time periods to wash out the possible initial noise effect. The results are given in Fig. 3. For four value of $\alpha$ we plot the average market price (left column), return (middle column), and the fundamentalists absolute wealth share accumulation (right column). The initial wealth share for both types of traders are equal $w_{1,0}=0.5$. Because of $m=0$, both the absolute and market wealth shares are the same.
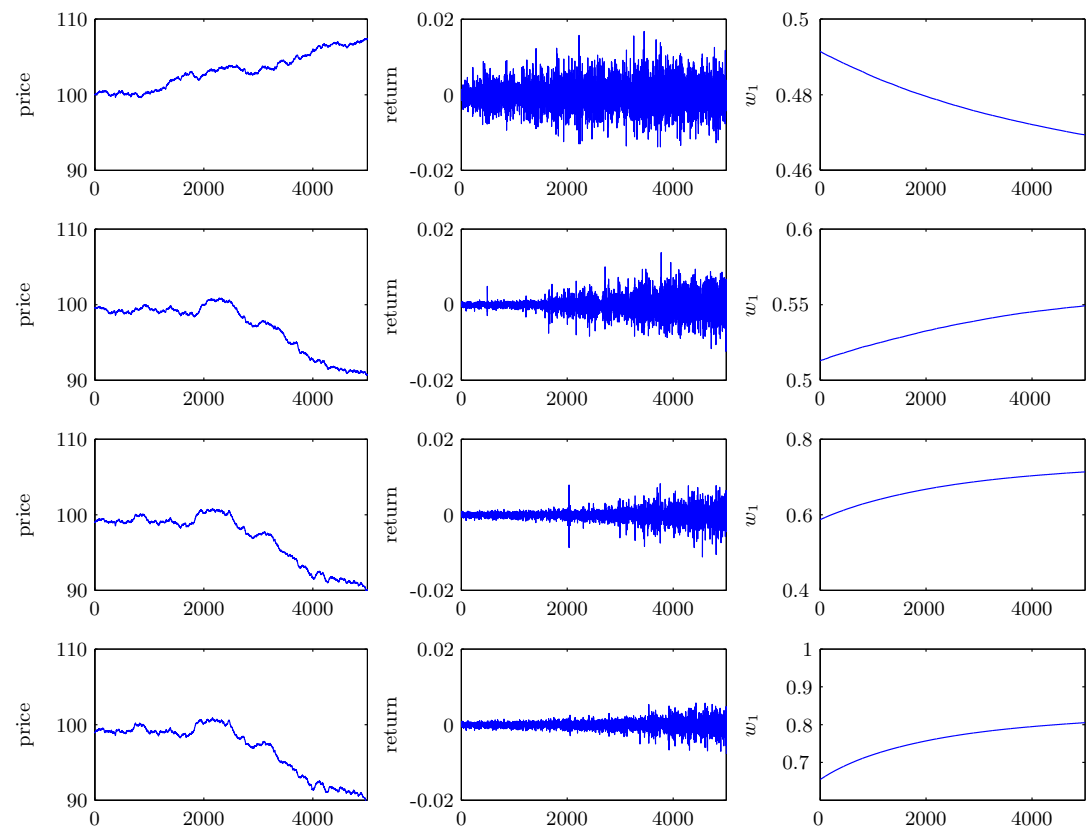

Fig. 3. Average Monte Carlo time series of market prices, returns, absolute wealth share and market wealth share of the fundamentalists with $\alpha=0,0.1,0.5$ and 1 .

\subsection{Dynamics of the Market Fraction $m$}

Intuitively it seems that the market price is partially determined by the market dominance of different players in the market. Therefore we would expect that the market fraction, measured by parameter $m$, will influence the market price and the consequent performance of fundamentalists and chartists.

Given that both $\alpha$ and $m$ have a similar impact on the local stability of the deterministic system (see He and $\mathrm{Li}[17]$ ), we can demonstrate that they play a similar role in terms of wealth accumulation. Again, by running one 
simulation over 20,000 time periods, Fig. 4 shows the absolute wealth share accumulations for the fundamentalists for three different values of $m=-0.95,0$ and 0.5 with $\alpha=0.5, \gamma=2, \mu=0.5, \delta=0.85, w_{1,0}=0.5$. In this case, the fundamentalists form their conditional expectation by taking an average of the latest market price and fundamental price. In all four cases, (i) the fundamentalists accumulate more wealth share than the trend followers in the long-run (an increase from $50 \%$ to about 70-75\%), however, the trend followers survive in the long-run and they can even accumulate more wealth share in the shortrun when they dominate the market (this is the case when $m=-0.95$, which corresponds to $97.5 \%$ of trend followers and $2.5 \%$ of the fundamentalists); (ii) the profitability of the fundamentalists improves as $m$ increases (i.e. as the market fraction of the fundamentalists increases). Essentially, we have shown that both $\alpha$ and $m$ have a similar effect on profitability and survivability for fundamentalists and trend followers. Comparison of Fig.2 and Fig. 4 indicates that the parameter $\alpha$ affects wealth accumulation more than the parameter $m$ does.

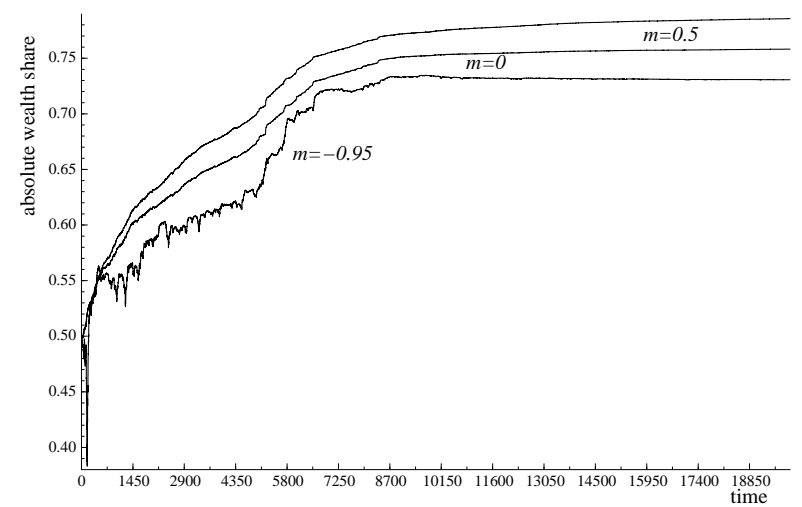

Fig. 4. Time series of the absolute wealth accumulation of the fundamentalists $w_{1, t}$ with $m=-0.95,0,0.5$ and $\alpha=0.5, \gamma=2, \mu=0.5, \delta=0.85, w_{1,0}=0.5$.

When the fundamentalists are naive traders (i.e. $\alpha=0$ and $E_{1, t}\left(P_{t+1}\right)=$ $\left.P_{t}\right)$ fundamental price doesn't influence how they form their conditional expectation. We choose

$$
\alpha=0, \gamma=1, \mu=0.4, \delta=0.85, w_{1,0}=0.5, m=-1,-0.5,0,0.5,1 .
$$

Again, for each set of parameters we run one simulation over 20,000 time periods such that the corresponding limiting behaviour becomes clear. Fig. 5 illustrates the absolute wealth share accumulations of the fundamentalists with different market fraction $m=-1,-0.5,0,0.5,1$, and keeping all the other conditions the same. They converge to different constant levels for different values of $m$ in the long-run. Note that, unlike the market price, the absolute 
wealth shares are independent from the market fraction $m$ and they are calculated for the given market price series. In particular, when $m= \pm 1$ the market price is affected only by one type of traders, but the absolute wealth share accumulations can still be calculated based on the market price. Fig. 5 shows that, overall, no one does significantly better by accumulating significant higher absolute wealth share than the others. For $m=1$ trend followers don't affect the market price. In this case, the long-run absolute wealth share accumulation of the fundamental trading strategy stays just above the average level, indicating that the trend followers will survive in long-run, although they have no impact on the market price and accumulate less absolute wealth share. For $m \neq 1$ trend followers do slightly better by accumulating a higher absolute wealth share. When $m=-1$ fundamentalists don't affect the market price. Under this scenario the trend followers accumulate more absolute wealth share in long-run. Overall, the profitability of the fundamentalists improves as $m$ increases (i.e. as their market population share increases). These results are further confirmed when we run Monte Carlo simulations. The results in Fig. 6 include the average market price, return, and absolute wealth share accumulation for the fundamentalists. The initial wealth share for both types of traders are equal $w_{1,0}=0.5$. For different values of $m$, the market wealth shares are different. It is also interesting to see that the average market price increases, rather than decreases in the first case, stochastically. Given the naive expectation of the fundamentalists this may be due to the trend chasing activity of the trend followers.

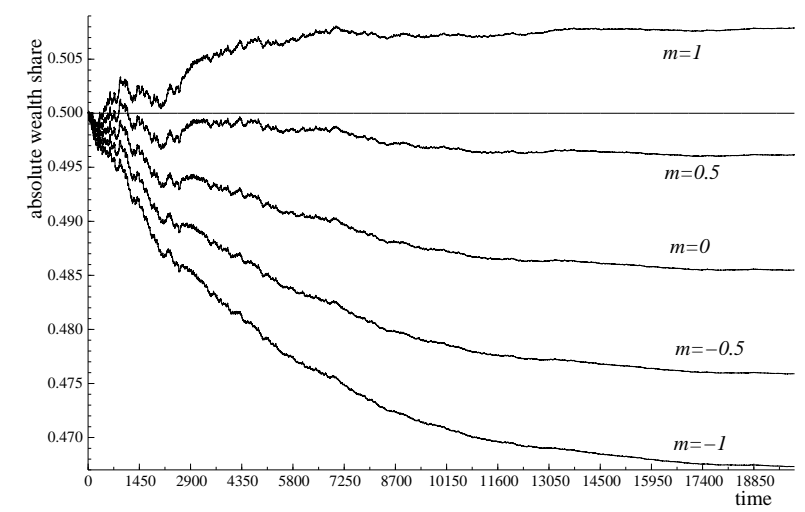

Fig. 5. Time series of the absolute wealth accumulation of the fundamentalists $w_{1, t}$ with $m=-1,-0.5,0,0.5,1$ and $\alpha=0, \gamma=1, \mu=0.4, \delta=0.85, w_{1,0}=0.5$.

The above analysis leads to the following implications for profitability and survivability:

- Although the trend followers have no information on the fundamental value they survive in the long-run and can even out-perform fundamentalists 

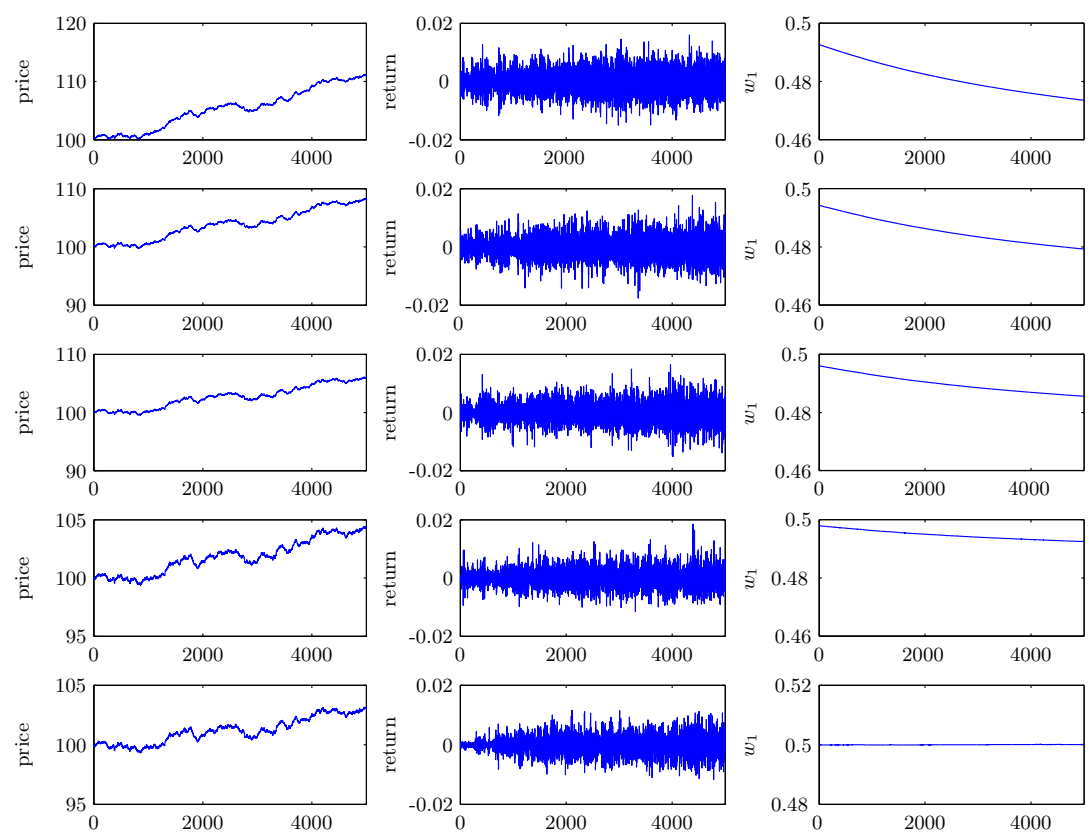

Fig. 6. Average Monte Carlo time series of market prices, returns and absolute wealth share of the fundamentalists with $\alpha=0, \gamma=1, \mu=0.4, \delta=0.85, w_{1,0}=$ 0.5 , and $m=-0.5$ (top row), 0 (second row), 0.5 (third row), 1(4-th row), -1(the last row).

in the short-run. This may be due to the learning mechanism they are engaged in.

- Fundamentalists' profitability increases as they become more confident in their estimates of fundamental value or they dominate the market.

- When the fundamentalists become naive traders, trend followers do better and they accumulate a higher wealth share. In addition, their profitability improves as their market population share increases.

The wealth share measures used in this paper compare the relative performance between two trading strategists. Survivability of the chartists is measured by their positive wealth share generated from their trading strategy. Overall, we have shown the short/long-run profitability for both the fundamental and trend following trading strategies, and the long-run survivability for the trend following strategy. This result partially verifies Friedman's argument that the chartists may do better in the short-run, but the market will be dominated by the fundamentalists in the long-run. However, in contrast to Friedman's argument, chartists do survive in the long-run due to the learning mechanism. 


\section{Conclusion}

In this study a market fractions model with heterogeneous traders in a simple asset-pricing and wealth dynamics framework is employed to investigate market dominance, profitability, and whether investors who follow fundamental and trend following strategies survive in short/long-run. Two measures of wealth are introduced to assess the connection between market dominance and wealth dynamics.

The conclusions drawn from the statistical analysis, based on Monte Carlo simulations, show that when the market is dominated by fundamentalists both their absolute and market wealth proportions increase significantly in the longrun. On the other hand, when trend followers dominate the market and the deviation between fundamental price and the trending price series is small, their wealth proportions fails to increase significantly. When the deviation is large trend followers' proportion of wealth increase.

The level of confidence fundamentalists have in the convergence of market price to fundamental value impacts upon trend followers wealth proportions. When they are less confident of convergence the market price can be driven away by trend followers and fundamentalists' wealth proportions decrease. Though, the trend followers' commensurate increase in wealth is relatively small. As fundamentalists become increasingly confident in convergence trend followers' wealth proportions can be reduced dramatically over the long-run. The key finding from our analysis which differs from what would be predicted by Friedman is that trend followers do survive in the long-run in all scenarios, while in the short-run they can out-perform fundamentalists which is consistent with the traditional view.

A potentially fruitful line for future research would be to explore the impact of information costs. In practice, information costs can be a non-trivial component of fundamental analysis. Integrating a range of plausible costs structures into a MF model could elucidate their affect on fundamentalists wealth share. In contrast, chartists' reliance on costless information and learning may explain their survival in the long-run.

\section{References}

1. Arnold, L. (1998), Random Dynamical Systems, Springer-Verlag, Berlin.

2. Arthur, W., J. Holland, B. LeBaron, R., Palmer, R. and P. Tayler (1997), Asset Pricing Under Endogeneous Expectations in an Artifical Stock Market, Economic Notes, 26(2), 297-330.

3. Brock, W., and C. Hommes (1997), A Rational Route to Randomness, Econometrica, 65, 1059-1095.

4. Brock, W., and C. Hommes (1998), Heterogeneous Beliefs and Routes to Chaos in a Simple Asset Pricing Model, Journal of Economic Dynamics and Control, $22,1235-1274$. 
5. Chan, L., and J. Lakonishok (2004), Value and Growth Investing: Review and Update, Financial Analysts Journal, 60, 71-86.

6. Chiarella, C. (1992), The Dynamics of Speculative Behaviour, Annals of Operations Research, 37, 101-123.

7. Chiarella, C., and X. He (2001), Asset Pricing and Wealth Dynamics under Heterogeneous Expectations, Quantitative Finance, 1, 509-526.

8. Chiarella, C., and X. He (2002), Heterogeneous Beliefs, Risk and Learning in a Simple Asset Pricing Model, Computational Economics, 19, 95-132.

9. Chiarella, C., and X. He (2003a), Dynamics of Beliefs and Learning under $\alpha_{l^{-}}$ processes - Heterogeneous Case, Journal of Economic Dynamics \& Control, 27, 503-531.

10. Chiarella, C., and X. He (2003b), Heterogeneous Beliefs, Risk and Learning in a Simple Asset Pricing Model with a Market Maker, Macroeconomic Dynamics, 7, 503-536.

11. Cochrane, J.H. (2001), Asset Pricing, Princeton University Press, Princeton.

12. Day, R. and W. Huang (1990), Bulls, Bears and Market Sheep, Journal of Economic Behaviour and Organization, 14, 299-329.

13. Ding, Z., C. Granger, and R. Engle (1993), A Long Memory Property of Stock market Returns and a New Model, Journal of Empirical Finance, 1, 83-106.

14. Friedman, M. (1953), The Case for Flexible Exchange Rate, in Essays in positive economics, Chicago, University of Chicago Press.

15. Gaunersdorfer, A. (2000), Endogenous Fluctuations in a Simple Asset Pricing Model with Heterogeneous Agents, Journal of Economic Dynamics and Control, $24,799-831$.

16. Haugen, R. (2003), The New Finance: Overreaction, Complexity and Uniqueness, Prentice Hall.

17. He X., and Y. Li (2007a), Heterogeneity, Convergence, and Autocorrelations, Quantitative Finance, in press.

18. He X., and Y. Li (2007b), Power-law behaviour, Heterogeneity, and Trend Chasing, Journal of Economic Dynamics and Control, 31, 3396-3426.

19. Hommes, C. (2002), Modeling the Stylized Facts in Finance Through Simple Nonlinear Adaptive Systems, Proceedings of National Academy of Science of the United States of America, 99, 7221-7228.

20. Hommes, C. (2006), Heterogeneous Agent Models in Economics and Finance, Handbook of Computational Economics, Volume 2, Edited by K.L. Judd and L. Tesfatsion, Elsevier Science.

21. LeBaron, B. (2000), Agent-based Computational Finance: Suggested Readings and Early Research, Journal of Economic Dynamics \& Control, 24, 679-702.

22. LeBaron, B. (2001), A Builder's Guide to Agent-based Financial Markets, Quantitative Finance, 1(2), 254-261.

23. LeBaron, B. (2002), Calibrating an Agent-based Financial Market to Macroeconomic Time Series, Technical report, Brandeis University, Waltham, MA.

24. LeBaron, B. (2006), Agent-based Computational Finance, Handbook of Computational Economics, Volume 2, Edited by K.L. Judd and L. Tesfatsion, Elsevier Science.

25. Levy, M., H. Levy, and S. Solomon (2000), Microscopic Simulation of Financial Markets, Academic Press, New York.

26. Li, Y., B. Donkers and B. Melenberg (2006a), Econometric Analysis of Microscopic Simulation Models, CentER Discussion Papers 2006-99, Tilburg University. Available at: $\langle$ http : //ssrn.com/abstract $=939518\rangle$. 
27. Li, Y., B. Donkers and B. Melenberg (2006b), The Nonparametric and Semiparametric Analysis of Microscopic Simulation Models, CentER Discussion Papers 2006-95, Tilburg University. Available at: $\langle$ http ://ssrn.com/abstract $=$ 939510 .

28. Lux, T. (1998), The Social-economic Dynamics of Speculative Markets: Interacting Agents, Chaos, and the Fat Tails of Return Distributions, Journal of Economic Behaviour \& Organization, Vol. 33, 143-165.

29. Lux, T., and M. Marchesi (1999), Scaling and Criticality in a Stochastic Multiagent Model of a Financial Markets, Nature, 397(11), 498-500.

30. Pagan, A. (1996), The Econometrics of Financial Markets, Journal of Empirical Finance, 3, 15-102.

31. Shiller, R. J. (2003), From Efficient Markets Theory to Behavioural Finance, Journal of Economic Perspectives, 17(1), 83-104.

32. Taylor, M., and H. Allen (1992), The Use of Technical Analysis in the Foreign Exchange Market, Journal of International Money and Finance, 11, 304-314. 